\title{
Factors influencing RhoA protein distribution in the nucleus
}

\author{
YUEYING LI, YONGCHANG CHEN and JIN XU
}

School of Medical Science and Laboratory Medicine, Jiangsu University, Zhenjiang, Jiangsu 212013, P.R. China

Received April 19, 2011; Accepted August 8, 2011

DOI: $10.3892 / \mathrm{mmr} .2011 .556$

\begin{abstract}
The aim of the present study was to observe the influence of various factors on the nuclear distribution of the RhoA protein in the SGC-7901 human gastric cancer cell line. Immunofluorescence microscopy was used to detect the localization of the RhoA protein, and Western blotting was used to determine the quantity of RhoA in the nucleus, cytosol and membrane. The results showed that $\mathrm{H}_{2} \mathrm{O}_{2}$-mediated damage and a lipopolysaccharide (LPS)-mediated inflammatory reaction caused the translocation of RhoA from the cytosol toward the nucleus. A P38 mitogen-activated protein kinase (MAPK) inhibitor effectively hindered the LPS-triggered translocation of RhoA into the nucleus at the initial stage. Furthermore, the microtubule-targeted anticancer drug Taxol triggered the translocation of RhoA from the nucleus toward the cytosol and membrane, and Lysophosphatidic acid (LPA) enhanced this translocation. A protein modification inhibitor and a nucleus export inhibitor had no obvious effect on RhoA distribution in the nucleus. The results revealed that the distribution of RhoA protein in the nucleus was influenced by factors related to cell activities but was not affected by the modification of the protein. The translocation of RhoA into the nucleus was not dependent on the active nuclear import system.
\end{abstract}

\section{Introduction}

RhoA, with a molecular mass of $21 \mathrm{kDa}$, is the most extensively studied member of the Rho GTPase family which belongs to the Ras superfamily of small G proteins. It has been reported to regulate many biological activities including the formation of stress fibers, gene transcription, membrane transport and focal adhesions and tumor progression (1-4). RhoA cycles between a GDP-bound inactive state and a GTP-bound active state (5). These two forms can be converted by GDP/GTP exchange or GTPase reactions. The former and latter reactions are regu-

Correspondence to: Professor Yongchang Chen, Department of Physiology, Jiangsu University, 301 XueFu Road, Zhenjiang, Jiangsu 212013, P.R. China

E-mail: ycchen54@ujs.edu.cn

Abbreviations: LPA, Lysophosphatidic acid; LMB, leptomycin B; LPS, lipopolysaccharide; LDH, Lactate dehydrogenase.

Key words: RhoA, nucleus, damage, inflammation, Taxol lated by GDP/GTP-exchange protein and GTPase-activating protein, respectively (6-8).

Recent research data have shown that various small G-proteins have cytoplasmic-nuclear dual distribution, and the nuclear translocation of small $\mathrm{G}$ protein is usually accompanied by the activation of the protein (9-12). For example, nuclear localized Ras protein exists in a GTP-bound form and this localization is possibly associated with malignancies of the cell (11). For a long time, an abundance of study data (13-15) have indicated that the RhoA protein is mainly distributed in the cytoplasm and on the membrane, but particularly in cytoplasm. However, our previous study (16) indicated that the distribution of RhoA in SGC-7901 cells was not only in the membrane and the cytoplasm but also in the nucleus. Recently, we substantiated the nuclear localization of RhoA in different cancer tissues and cell lines (17). Regarding the effect of the activation of RhoA on its localization and translocation in cells, our previous study (16) revealed that when the activity of RhoA increased, the distribution of RhoA on the membrane and in the nucleus also increased, which indicates that the nuclear distribution of RhoA has a potential role in regulating cellular activity.

To further study the mechanism of the nuclear distribution of RhoA and the function of the RhoA protein in the nucleus, this study was designed to observe the effect of several treatments including protein modification, cellular damage, inflammation and Taxol treatment on the nuclear distribution of RhoA.

\section{Materials and methods}

Cell lines. Human gastric cancer cell line SGC-7901 was obtained from the Institute of Cell Biology of the Chinese Academy of Sciences (Shanghai, China).

Reagents. Dulbecco's modified Eagle's medium (DMEM) was purchased from Gibco (Grand Island, NY, USA). Newborn calf serum (NBCS) was from Minhai Bio-Engineering $\mathrm{C}$ (Lanzhou, China). Mouse monoclonal antibody against RhoA was from Santa Cruz Biotechnology (Santa Cruz, CA, USA). The antibody against glyceraldehyde phosphate dehydrogenase (GAPDH) was from Kangcheng (China). The horseradish peroxidase (HRP)-conjugated secondary antibody was from Jackson ImmunoResearch Laboratories (West Grove, PA, USA). Simvastatin, 5-aza-2'-deoxycytidine (5-AzaCdr), dexamethasone, lipopolysaccharide (LPS), SB203580 and leptomycin B (LMB) were from Sigma (St. Louis, MO, USA). Electrochemiluminescence (ECL) reagents were from 
Amersham Biosciences (Buckinghamshire, UK). Taxol was from Calbiochem. The reagent for determining Lactate dehydrogenase (LDH) was from the Jiancheng Biology Engineering Institute (Nanjing, China).

Cell culture. The cells were cultured in DMEM supplemented with $10 \% \mathrm{NBCS}$ and maintained at $37^{\circ} \mathrm{C}$ in a humidified atmosphere of $5 \% \mathrm{CO}_{2}$. The medium was replaced every 2 days, and the cells were subcultured at confluence.

Immunofluorescence microscopy. The cells grown on coverslips were fixed with freshly prepared paraformaldehyde $(40 \mathrm{~g} / \mathrm{l}$ in PBS) for $30 \mathrm{~min}$. After being penetrated with $30 \mathrm{ml} / 1$ Triton $\mathrm{X}-100$ and blocked with $30 \mathrm{~g} / \mathrm{l}$ bovine serum albumin (BSA), the cells were incubated with the primary antibody at $4^{\circ} \mathrm{C}$ overnight $(\mathrm{o} / \mathrm{n})$ and then with fluorescein isothiocyanate (FITC) or a tetrarhodamine isothiocyanate (TRITC)-conjugated second antibody for $1 \mathrm{~h}$ at room temperature (RT), with three washes after each incubation. The distribution of the target protein of the cells was analyzed by fluorescence microscopy.

Preparation of the nuclear protein. According to a previously described method (18), 1-3 million cells were washed with $10 \mathrm{ml}$ of Tris-buffered saline (TBS) and pelleted by centrifugation at $1500 \mathrm{x} \mathrm{g}$ for $5 \mathrm{~min}$. The pellet was resuspended in $1 \mathrm{ml}$ of TBS, transferred into an Eppendorf tube and pelleted again by spinning for $15 \mathrm{sec}$ in a microcentrifuge. The cell pellet was resuspended in $400 \mu 1$ of cold buffer A $(10 \mathrm{mM}$ HEPES pH 7.9, $10 \mathrm{mM} \mathrm{KCl,} \mathrm{0.1} \mathrm{mM} \mathrm{EDTA,} 0.1 \mathrm{mM}$ EGTA, $1 \mathrm{mM}$ DTT and $0.5 \mathrm{mM}$ PMSF) by gentle pipetting. The cells were allowed to swell on ice for $15 \mathrm{~min}$, after which $25 \mu \mathrm{l}$ of NP-40 (0.5\%) was added and the tube was thoroughly mixed for $10 \mathrm{sec}$. The homogenate was centrifuged for $30 \mathrm{sec}$ and the supernatant containing cytoplasm and RNA was saved for future use if needed. The nuclear pellet was resuspended in $50 \mu \mathrm{l}$ of ice-cold buffer C (20 mM HEPES pH 7.9, $400 \mathrm{mM}$ $\mathrm{NaCl}, 1 \mathrm{mM}$ each of DTT, EDTA, EGTA and PMSF). The tube was mixed thoroughly and placed on a rotatory shaker for $15 \mathrm{~min}$. The nuclear extract was centrifuged at $11,000 \mathrm{x} \mathrm{g}$ for $5 \mathrm{~min}$ (microcentrifuge). The supernatant containing the proteins from the nuclear extract was removed carefully to a fresh tube. The protein was measured in the nuclear extract, which was then aliquoted in several tubes and stored at $-80^{\circ} \mathrm{C}$.

Western blotting. Sample proteins were run on $10 \%$ SDS-polyacrylamide gels, and were blotted onto polyvinyl difluoride (PVDF) membranes. The PVDF membrane was blocked with 3\% (w/v) BSA in TBS-T for $1 \mathrm{~h}$ at RT. Membranes were incubated with the primary antibody at $4{ }^{\circ} \mathrm{Co} / \mathrm{n}$, and with the secondary antibody for $1 \mathrm{~h}$ at RT, with three washes after each incubation. Electrochemiluminescence reagents were used to show the positive bands on the membrane. The bands on film were analyzed with GeneSnap/Gene Tool software from Syngene (Cambridge, UK).

Measure of LDH activity. The total LDH activity was measured by colorimetry.

Statistical analysis. Data are expressed as the mean \pm SE. Statistical analysis was performed using SPSS 13.0 edition program for ANOVA with the Scheffè multiple comparison test. A probability value of $<0.05$ was considered statistically significant.

\section{Results}

Effect of RhoA modification on its distribution within the nucleus. Research data has indicated that modification of small $\mathrm{G}$ proteins has an important effect on their distribution in the nucleus. In order to elucidate whether the modification of the RhoA protein has an effect on its nuclear distribution, inhibition of the modification of prenylation and methylation was achieved by applying the prenyltransferase inhibitor, simvastatin, and the methyltransferase inhibitor, 5-Aza-Cdr, respectively. The effect of the inhibition was observed by immunofluorescence microscopy. The results showed that neither the inhibition of prenylation nor the inhibition of methylation had any significant effect on the distribution of RhoA within the nucleus in the human gastric cancer cell line SGC-7901 (Fig. 1), which suggested that the cytoplasmnucleus transfer of RhoA was not affected by modifications to the RhoA protein.

Distribution of RhoA within the nucleus in damaged cells. To observe the distribution of RhoA upon cellular damage, human gastric cancer cell line SGC-7901 was treated with $\mathrm{H}_{2} \mathrm{O}_{2}$. Damage to the cells was confirmed by detecting $\mathrm{LDH}$ activity in the culture medium (Fig. 2A). Immunofluorescence microscopy showed that the amount of RhoA increased in the damaged cells, and more RhoA was translocalized to the nucleus (Fig. 2B).

Effect of Taxol on RhoA distribution within the nucleus. Taxol is an antitumor drug targeting the microtubule structure of tumor cells. To observe the effect of microtubule structure dynamics on the distribution of RhoA, human gastric cancer cell line SGC-7901 was treated with Taxol, and Taxol combined with Lysophosphatidic acid (LPA). Immunofluorescence microscopy showed that Taxol triggered the translocation of RhoA from the nucleus towards the cytosol and membrane, and LPA enhanced this translocation (Fig. 3). These results suggest that the distribution of RhoA is related to the microtubule structure in cells.

Effect of LPS on the distribution of RhoA within the nucleus. To observe the inflammatory effect on the distribution of RhoA, human gastric cancer cell line SGC-7901 was treated with LPS to mimic an inflammatory effect. Results of the immunofluorescence staining (Fig. 4A) and Western blotting (Fig. 4B and C) showed that, in LPS-treated cells, the nuclear RhoA increased significantly, the translocation of RhoA from the cytosol toward the nucleus was obvious, and some cells even showed that without RhoA distribution in the cytosol, the nucleus moved largely towards one side of the cell, resembling a 'shining ring'.

Effect of P38 mitogen-activated protein kinase (MAPK) inhibitor (SB203580) on the translocation of RhoA within the nucleus triggered by LPS. To further confirm the inflammatory effect on RhoA distribution, human gastric cancer cell line SGC-7901 was treated with a P38 MAPK inhibitor 


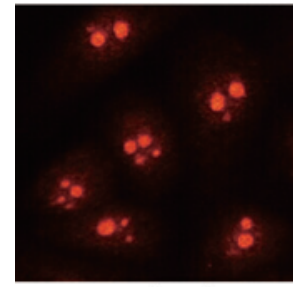

control

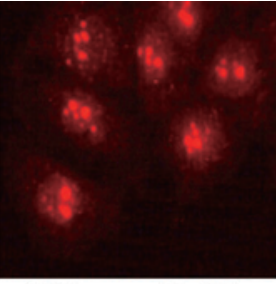

Simvastatin

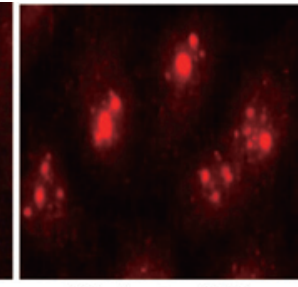

5-Aza-Cdr

Figure 1. Effect of RhoA modification on its distribution within the nucleus. Human gastric cancer cell line SGC-7901 was treated with prenyltransferase inhibitor simvastatin $(0.125 \mu \mathrm{l} / \mathrm{ml})$ and methyltransferase inhibitor 5-Aza-Cdr $(0.25 \mu \mathrm{l} / \mathrm{ml})$, respectively, for $24 \mathrm{~h}$, and the cells were then immunofluorescently stained with a specific mAb against RhoA. The results revealed that the inhibition of neither prenylation nor methylation had any significant effect on the distribution of RhoA within the nucleus (x400).

A

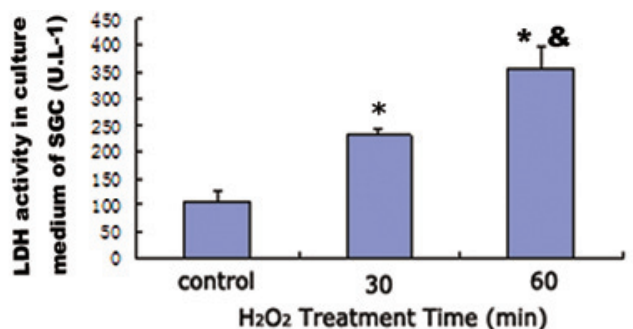

B

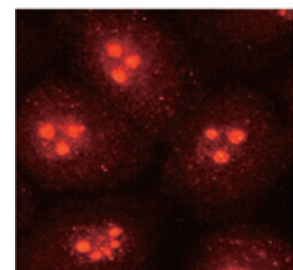

control

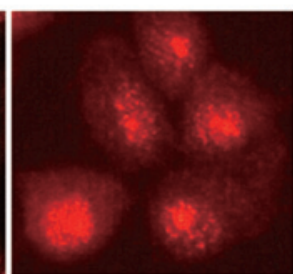

$\mathrm{H}_{2} \mathrm{O}_{2} 30 \mathrm{~min}$

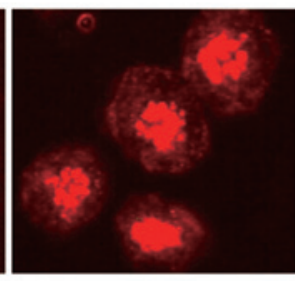

$\mathrm{H}_{2} \mathrm{O}_{2} 60 \mathrm{~min}$
Figure 2. Distribution of RhoA within the nucleus in damaged cells. Human gastric cancer cell line SGC-7901 was treated with $1 \mathrm{M} \mathrm{H}_{2} \mathrm{O}_{2}$, and the $\mathrm{LDH}$ activity in the culture medium was measured at different time points $(0,30$ and $60 \mathrm{~min}$ ). (A) LDH activity was analyzed by SPSS 13.0 Tool software. Each bar represents the mean $\pm \mathrm{SE}$ obtained from five independent experiments. Bar 1, expression of LDH activity of the control group; SGC-7901 cells were not treated with $\mathrm{H}_{2} \mathrm{O}_{2}$; bar 2, expression of LDH activity of SGC7901 cells treated with $\mathrm{H}_{2} \mathrm{O}_{2}$ for $30 \mathrm{~min}$; bar 3, expression of $\mathrm{LDH}$ activity of SGC-7901 cells treated with $\mathrm{H}_{2} \mathrm{O}_{2}$ for 60 min. ${ }^{*} \mathrm{P}<0.05$, compared with bar 1 ; ${ }^{\&} \mathrm{P}<0.05$, compared with bar 2. (B) Results of the immunofluorescent staining with a specific mAb against RhoA, indicating that the total amount of RhoA and nuclear RhoA increased significantly as cells underwent damage at 30 and 60 min after $\mathrm{H}_{2} \mathrm{O}_{2}$ treatment (x400).

(SB203580) $1 \mathrm{~h}$ before LPS treatment. Immunofluorescence microscopy showed that after $3 \mathrm{~h}$, the P38 MAPK inhibitor SB203580 effectively hindered the LPS-triggered translocation of RhoA into the nucleus, which was represented by increased distribution of RhoA within the cytosol, resembling 'dispersive sand'; while after a prolonged treatment of LPS, significant translocation of RhoA from the cytosol into the nucleus was observed (Fig. 5).

Effect of nucleus-export inhibitor on the distribution of RhoA within the nucleus. To study the molecular mechanism of RhoA transportation to the nucleus, human gastric cancer cell line SGC-7901 was treated with the nucleus export inhibitor, lepto-

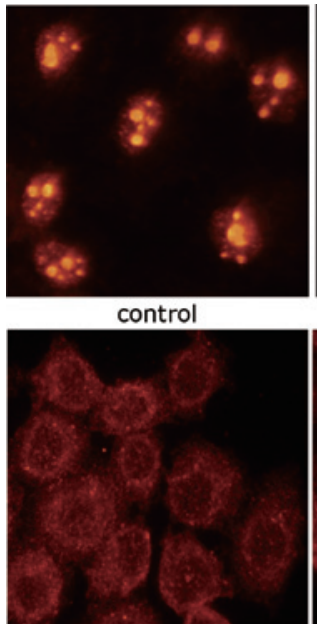

Taxol $50 \mu \mathrm{g} / \mathrm{ml}$

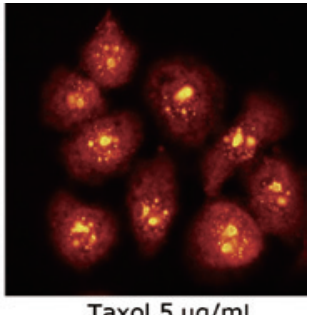

Taxol $5 \mu \mathrm{g} / \mathrm{ml}$

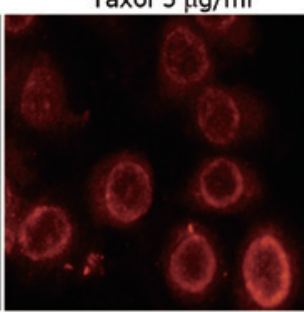

Taxol + LPA

Figure 3. Effect of Taxol on RhoA distribution within the nucleus. Human gastric cancer cell line SGC-7901 was treated with 5 or $50 \mu \mathrm{g} / \mathrm{ml}$ Taxol or $5 \mu \mathrm{g} / \mathrm{ml}$ Taxol combined with $1 \mu \mathrm{M}$ LPA, respectively, for $6 \mathrm{~h}$. Results of the immunofluorescent staining with a specific mAb against RhoA showed that Taxol triggered the translocation of RhoA from the nucleus toward the cytosol and the membrane, and that LPA enhanced this translocation (x400).

A

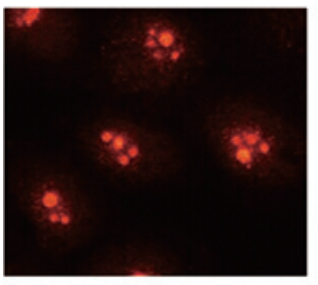

control

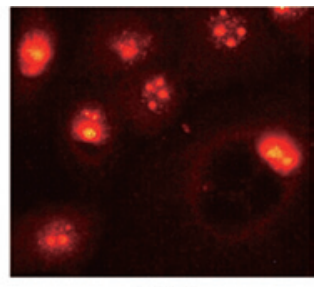

LPS
$\mathbf{B}$

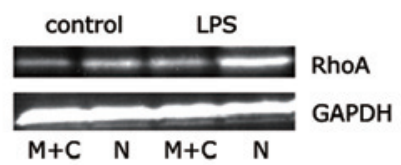

C

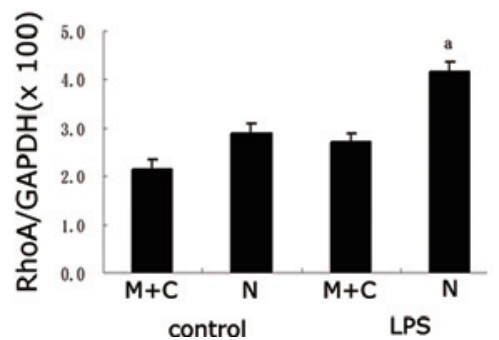

Figure 4. Effect of LPS on the distribution of RhoA within the nucleus Human gastric cancer cell line SGC-7901 was treated with LPS $(1 \mu \mathrm{g} / \mathrm{ml})$ for $12 \mathrm{~h}$. (A) Results of the immunofluorescent staining with a specific $\mathrm{mAb}$ against RhoA revealed that the translocation of RhoA from the cytosol toward the nucleus was significant. In some cells even showing a lack of RhoA in the cytosol, the nucleus moved largely towards one side of the cell, resembling a 'shining ring' (x400). (B) Two groups of SGC-7901 cells were lysed, and the membrane and cytosol $(\mathrm{M}+\mathrm{C})$, and the nuclear $(\mathrm{N})$ fractions were obtained. Expression of RhoA and GAPDH in each fraction was detected by Western blotting. (C) Western blotting results were analyzed by SPSS 13.0 Tool software, and the volume ratio of RhoA/GAPDH input was calculated and presented. Each bar represents the mean $\pm \mathrm{SE}$ obtained from three independent experiments. Bar 1, expression of RhoA in the $\mathrm{M}+\mathrm{C}$ fraction of the control group; SGC-7901 cells were not treated with LPS; bar 2, expression of RhoA in the $\mathrm{N}$ fraction of the control group; bar 3, expression of RhoA in the M + C fraction of SGC-7901 cells treated with LPS for $12 \mathrm{~h}$; bar 4, expression of RhoA in the $\mathrm{N}$ fraction of SGC-7901 cells treated with LPS for $12 \mathrm{~h}$. ${ }^{\mathrm{a}} \mathrm{P}<0.05$, compared with bar 2 . 

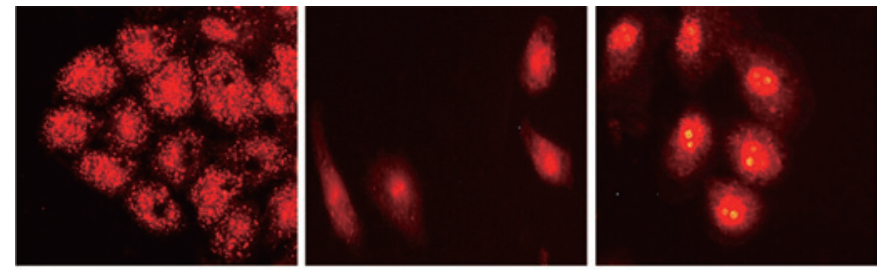

P38 $1 h+$ LPS $3 h$ P38 $1 h+$ LPS $6 h$ P38 $1 h+$ LPS $12 h$

Figure 5. Effect of the P38 MAPK inhibitor (SB203580) on the translocation of RhoA within the nucleus triggered by LPS. Human gastric cancer cell line SGC-7901 was cultured in serum-free medium for $24 \mathrm{~h}$, and the cells were then divided into 4 groups; one group was set as the control and the other 3 groups were treated with $2 \mu \mathrm{g} / \mathrm{ml}$ P38 MAPK inhibitor (SB203580) for $1 \mathrm{~h}$ before treatment with LPS $(1 \mu \mathrm{g} / \mathrm{ml})$. The samples were collected at 3,6 and $12 \mathrm{~h}$, respectively, and immunofluorescent staining was carried out with a specific $\mathrm{mAb}$ against RhoA. The results revealed that after $3 \mathrm{~h}$, the inhibitor SB203580 effectively hindered the LPS-triggered translocation of RhoA into the nucleus, which was represented by increased distribution of RhoA within the cytosol, resembling 'dispersive sand'; while after a more prolonged treatment of LPS, significant translocation of RhoA from the cytosol into the nucleus was observed $(x 400)$.

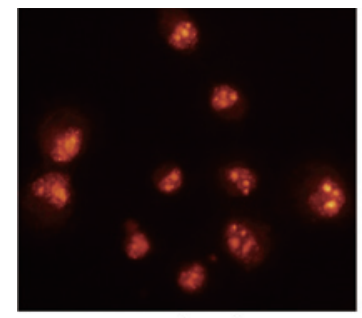

control

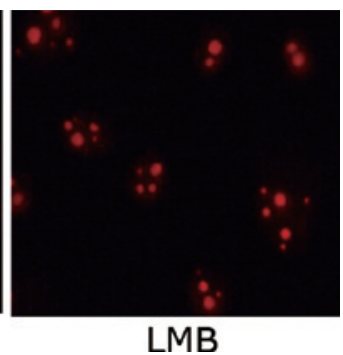

LMB
Figure 6. Effect of a nucleus-export inhibitor on the distribution of RhoA within the nucleus. Human gastric cancer cell line SGC-7901 was treated with nucleus export-inhibitor leptomycin B (LMB, $20 \mathrm{ng} / \mathrm{ml})$ for $12 \mathrm{~h}$. The results of the immunofluorescent staining with a specific $\mathrm{mAb}$ against RhoA reflected the existence of RhoA within the nucleus, indicating no significant effect of the nucleus export-inhibitor on RhoA distribution within the nucleus (x400)

mycin B (LMB). Immunofluorescence microscopy showed that RhoA still existed within the nuclei in LMB-treated cells, indicating no significant effect of LMB on RhoA distribution within the nucleus (Fig. 6).

\section{Discussion}

Research data have shown that modification of some small G-proteins is related to the distribution of the proteins within cells. For example, members of the Ras superfamily are subject to post-translational modifications and the spectrum of modifications depends on the composition of the carboxyl terminus. In the case of Ha-Ras, the protein is subject to prenylation, proteolysis, carboxylmethylation and S-acylation/ palmitoylation (19). The prenylated Ha-Ras in the cytoplasm is then transferred by the endomembrane system to the plasma membrane (20). Research data also suggest that prenylated Rab acceptor protein (PRA1) binds prenylated small G-proteins to act as an escort protein for the GTPases (21). Prenylation is followed by proteolysis of the carboxyl-terminal tripeptide and methylation of the newly generated carboxyl-terminal amino acid in the endoplasmic reticulum (ER) $(19,21)$. Without prenylation, the subsequent processing events do not occur, and the Ras proteins remain soluble $(19,21)$. Statins may inhibit the prenylation of protein, disorder or destroy the cell framework, and remodel activation of many nuclear transfer genes by the signaling pathways of JNK/ SAPK and P38 MARK. Our study revealed that neither the inhibition of prenylation nor the inhibition of methylation had any significant effect on the distribution of RhoA within the nucleus. This suggests that the cytoplasmic-nuclear transfer of RhoA is not affected by modifications.

Taxol, an antimicrotubule agent, has the ability to destroy karyokinesis of cancer cells by strengthening the aggregation of canaliculus protein and inhibiting division, as well as the ability to inhibit and kill cancer cells (22-24). It has previously been shown to be very effective against ovarian and breast cancer, and recent clinical trials have shown that Taxol may also be a useful agent in the treatment of non-small cell lung cancer, head and neck cancer and various other forms of the disease (25). Taxol treatment may cause defects in mitotic spindle assembly, chromosome segregation and cell division. The ability of Taxol to inhibit spindle function is generally attributed to its suppression of microtubule dynamics. Our results showed that Taxol hindered the translocation of RhoA into the nucleus. On one hand, this suggests that the distribution of RhoA in the nucleus is related to the microtubule structure in the cells. On the other hand, due to the extreme importance of microtubules in the process of mitosis and tubulin being one of the most important targets for new anticancer drugs (26), RhoA may be a novel target for the diagnosis and therapy of tumors.

Lipopolysaccharide (LPS) is a primary factor which induces inflammatory diseases. When stimulated by bacterial LPS, many intracellular signaling pathways are activated, leading to the generation of nuclear factor $N F-\kappa B$, which in turn promotes pro-inflammatory cytokine production and release (27). Caplan et al (28) reported the up-regulation of MD-2, one of the bacterial LPS co-receptors, in patients with neonatal necrotizing enterocolitis. Inflammatory mediators released during acute and chronic diseases can activate multiple intracellular signals inducing cascades of the MAPK signaling transduction pathway. MAPK plays a significant role in the recruitment of leukocytes to the sites of inflammation (29). The present study showed that LPS promoted the translocation of RhoA from the cytosol toward the nucleus, and that a P38 MAPK inhibitor effectively hindered the LPS-triggered translocation of RhoA into the nucleus. This suggests that nuclear RhoA may play various roles in the inflammatory reaction. The possible mechanism still requires further elucidation.

This study also confirmed that more RhoA was distributed in the nucleus of damaged cells. Research data have indicated that when the cell encounters oxidation stress damage, expression of some proteins may increase, and the proteins may be rapidly transported into the nucleus to congregate there (30). Generally, stimuli promoting protein expression are also able to promote their intracellular movement, most of which are cytoplasm-to-nucleus translocations (31). In response to stress conditions or other stimuli, such as hypoxia, hyperoxia, mechanical stress, cAMP, hypotonic media, vasoactive agents and inflammation, several cell types release ATP via either a non-lytic or a lytic mechanism from necrosis and 
apoptosis, reaching high local concentrations $(32,33)$. High ATP-mediated metabolic stimuli may induce quick cytoplasm to nucleus translocation of the protein. On the other hand, when the tissue cells in the body undergo pathogeny, they induce certain inflammatoty factors or media, such as TNF- $\alpha$ (34). These inflammatory and damage factors are also likely to induce the translocation of RhoA into the nucleus. However, the exact mechanism which induces the nuclear distribution of RhoA is still unknown and warrants further study.

In order to enter the nucleus, proteins larger than $\sim 60 \mathrm{kDa}$ generally require a specific nuclear localization signal (NLS) (35). Protein molecules can bind with transporting protein such as importin via NLS and enter the nucleus through an ATP-dependent process (36). However, the molecular mass of RhoA is $21 \mathrm{kDa}$ and there is no NLS in its protein structure. Moreover, treatment with a nucleus-export inhibitor had no effect on the nuclear distribution of RhoA. Thus, we propose that the molecular mechanism of RhoA protein transportation into the nucleus was not through an active transportation pathway, but through a diffusion pathway.

\section{Acknowledgements}

This study was supported by the Natural Science Foundation of Jiangsu Province (no. BK2007091) and the Natural Science Fund for Colleges and Universities in Jiangsu Province, (no. 08KJD180007).

\section{References}

1. Price LS and Collard JG: Regulation of the cytoskeleton by Rho-family GTPases: implications for tumour cell invasion. Semin Cancer Biol 11: 167-173, 2001.

2. Sotiropoulos A, Gineitis D, Copeland J and Treisman R: Signal regulated activation of serum response factor is mediated by changes in actin dynamics. Cell 98: 159-169, 1999.

3. Kjoller L and Hall A: Signaling to Rho GTPases. Exp Cell Res 253: 166-179, 1999.

4. Narumiya S: The small GTPase Rho: cellular functions and signal transduction. J Biochem 120: 215-228, 1996.

5. Bar-Sagi D and Hall A: Ras and Rho GTPases: a family reunion. Cell 103: 227-238, 2000.

6. Bourne HR, Sanders DA and McCormick F: The GTPase superfamily: a conserved switch for diverse cell functions. Nature 348 : 125-132, 1990.

7. Hall A: The cellular functions of small GTP-binding proteins Science 249: 635-640, 1990.

8. Takai Y, Kaibuchi K, Kikuchi A and Kawata M: Small GTP-binding proteins. Int Rev Cytol 133: 187-230, 1992.

9. Moore MS and Blogel G: The GTP-binding protein Ran/TC4 is required for protein import into the nucleus. Nature 365: 661-663, 1993.

10. Moore JD: The Ran-GTPase and cell-cycle control. Bioessays 23: 77-85, 2001.

11. Mitra RS, Zhang Z, Henson BS, Kurnit DM, Carey TE and D'Silva NJ: Rap1A and rap1B ras-family proteins are prominently expressed in the nucleus of squamous carcinomas: nucleus traslocation of GTP-bound active form. Oncogene 22: 6243-6256, 2003.

12. Wurzer G, Mosgoeller W, Chabicovsky M, Cerni C and Wesierska-Gadek J: Nucleus Ras: unexpected subcellular distribution of oncogenic forms. Cell Biochem Suppl 36: 1-11, 2001.
13. Yonemura S, Hirao-Minakuchi K and Nishimura Y: Rho localization in cells and tissues. Exp Cell Res 295: 300-314, 2004.

14. Hoffman GR, Nassar N and Cerione RA: Structure of the Rho family GTP binding protein Cdc42 in complex with the multifunctional regulator RhoGDI. Cell 100: 345-356, 2000.

15. Adamson P, Paterson HF and Hall A: Intracellular localization of the P21rho proteins. Cell Biol 119: 617-627, 1992.

16. Yan Tao, Yong-Chang Chen and Yue-Ying Li: Localization and translocation of RhoA protein in human gastric cancer cell line SGC-7901. World J Gastroenterol 14: 1175-1181, 2008.

17. Li Y, Chen Y, Tao Y, Xu J and Chen M: RhoA protein is generally distributed in the nuclei of cancer cells. Oncol Rep 24: 1005-1009, 2010.

18. Lahiri DK and Ge Y. Electrophoretic mobility shift assay for the detection of specific DNA-protein complex in nuclear extracts from the cultured cells and frozen autopsy human brain tissue. Brain Res Protoc 5: 257-265, 2000.

19. Zhang FL and Casey PJ: Protein prenylation: molecular mechanisms and functional consequences. Annu Rev Biochem 65: 241-269, 1996.

20. Figueroa C, Taylor J and Vojtek AB: Prenylated Rab acceptor protein is a receptor for prenylated small GTPases. Biol Chem 276: 28219-28225, 2001.

21. Gelb MH: Protein prenylation, etcetera: signal transduction in two dimensions. Science 275: 1750-1751, 1997.

22. Bissery MC, Guénard D, Guéritte-Voegelein F and Lavelle F: Experimental antitumor activity of taxotere (RP 56976, NSC 628503), a Taxol analogue. Cancer Res 51: 4845-4852, 1991.

23. Dunn GA, Zicha D and Fraylich PE: Rapid, microtubule-dependent fluctuations of the cell margin. Cell Sci 110: 3091-3098, 1997.

24. Stracke ML, Soroush M, Liotta LA and Schiffmann E: Cytoskeletal agents inhibit motility and adherence of human tumor cells. Kidney Int 43: 151-157, 1993

25. Suffness M and Wall ME: Taxol. In: Science and Applications. Suffness M (ed). CRC Press Inc, Boca Raton, FL, pp1-448, 1995.

26. Shang H, Pan L, Yang S, Chen H and Cheng MS: [Progress in the study of tubulin inhibitors]. Yao Xue Xue Bao 45: 1078-1088, 2010 (In Chinese).

27. Akira S and Takeda K: Toll-like receptor signalling. Nat Rev Immunol 4: 499-511, 2004

28. Caplan MS, Simon D and Jilling T: The role of PAF, TLR, and the inflammatory response in neonatal necrotizing enterocolitis. Semin Pediatr Surg 14: 145-151, 2005.

29. Li Gang, Li Jieshou, Qi Xiaoping: p38 MAPK signalling cascades and inflammatory disease. Journal of Medical Postgraduates (in Chinese) 17: 737-740, 2004

30. Wei SJ, Botero A, Hirota K, Bradbury CM, Markovina S, Laszlo A, Spitz DR, Goswami PC, Yodoi J and Gius D: Thioredoxin nucleus translocation and interaction with redox factor-1 activates the activator protor- 1 transcription factor in response to ionizing radiation. Cancer Res 60: 6688-6695, 2000.

31. Tell G, Damante G, Caldwell D and Kelley MR: The intracellular localization of APE1/Ref-1: more than a passive phenomenon? Antioxid Redox Signal 7: 367-384, 2005.

32. Bowler WB, Littlewood-Evans A, Bilbe G, Gallagher JA and Dixon CJ: P2Y2 receptors are expressed by human osteoclasts of giant cell tumor but do not mediate ATP-induced bone resorption. Bone 22: 195-200, 1998

33. Ostrom RS, Gregorian C and Insel PA: Cellular release of and response to ATP as key determinants of the set-point of signal transduction pathways. Biol Chem 275: 11735-11739, 2000.

34. Shaw PJ, Ganey PE and Roth RA: Tumor necrosis factor alpha is a proximal mediator of synergistic hepatotoxicity from trovafloxacin/lipopolysaccharide coexposure. Pharmacol Exp Ther 328: 62-68, 2009.

35. Moroianu J: Molecular mechanisms of nucleus protein transport. Crit Rev Eukaryot Gene Expr 7: 61-72, 1997.

36. Friedrich B, Quensel C, Sommer T, Hartmann E and Kohler M: Nucleus localization signal and protein context both mediate importin alpha specificity of nucleus import substrates. Mol Cell Biol 26: 8697-8709, 2006. 